\title{
Quantum information
}

\section{Vlatko Vedral: Decoding reality: The universe as quantum information. Oxford: Oxford University Press, 2010, 218pp, \$15.09 HB \\ Christopher Fuchs: Coming of age with quantum information: Notes on a Paulian idea. Cambridge: Cambridge University Press, 2011, 533pp, \$70.00 HB}

\section{Fedde Benedictus}

Published online: 29 November 2011

(C) The Author(s) 2011. This article is published with open access at Springerlink.com

In the beginning of the twentieth century, humanity's view of the world went through a sea change. Not only did Einstein's relativity theories revolutionize the way we gaze at the heavens, Quantum Theory drastically altered our view of the microscopic. In Quantum Theory, particles are no longer the infinitely small, perfect spheres of the classical worldview of Newton and Boltzmann. In the new theory, matter is described in terms of a wave function. It is only when a measurement is made that particles appear. The existence of the wave function implies the uncertainty principle: the position and momentum of a particle described by a wave function can never be precisely defined at the same time.

This is about where the consensus ends, for physicists cannot agree on what this wave function and the consequent uncertainty principle imply for our reality. This disagreement has divided scientists into several camps. A Copenhagen-like interpretation abides to the positivist principle that we should not make statements about the unobservable. In this case, it is meaningless to discuss the wave function, as it cannot be measured.

Many Copenhagen-minded physicists hold that there must somehow be a transition from the realm of the quantum to the classical realm, as we observe no uncertainty. For this transition to take place, the mechanism of "wave function collapse" is often assumed. When a particle is observed, its wave function collapses onto a Dirac Delta function, thereby giving rise to a "classical" system. The position of the particle in this resulting classical system is determined by the form of the wave function. So, what is happening before our observation? Is the substance of a particle somehow "smeared out" over a finite region in space? Or does the particle have a definite position, and the uncertainty principle is merely a manifestation of our ignorance? In contrast to the Copenhagen followers-who held that this

\footnotetext{
F. Benedictus $(\bowtie)$

Institute for History and Foundations of Science, P. O. Box 80.000, 3508, TA,

Utrecht, The Netherlands

e-mail: f.j.benedictus@uu.nl
} 
question is meaningless-the American physicist David Bohm suggested that particles have a precise location and momentum at all times, so there is a kind of mechanics underlying that of the quantum-this proposal became known as "Bohmian mechanics."

During and after World War II, there was an increasing interest in methods of secure communication. Because of this interest, the branch of applied mathematics known as Information Theory received growing attention. The year 1948, in which Claude Shannon published his famous article "A Mathematical Theory of Communication," is generally regarded as the year in which modern information theory was born. According to this theory, the smallest amount of information is the bit. "Bit" is a portmanteau formed by the words "binary" and "digit," which tells us that at this most fundamental level, all information can be reduced to zeros and ones.

With the advent of quantum theory, the concept of information too was significantly changed. The material carriers of the bits in information theory were henceforth described in terms of their own wave function. This had a major consequence: a bit was no longer restricted to being either a zero or a one, but could be in a "superposition" of both. In 1982, Richard Feynman was the first to speculate on the application of such quantum effects in a computer.

Ever since, the field of quantum information theory has been one of rapid development. The fact that quantum bits can be in several states at the same time makes parallel calculations possible, giving rise to what is known as the "quantum speedup" of computers. So far, this speedup has only been realized in a system consisting of a few qubits-but the results are promising.

This promise has not only led to a wave of governmental funding and stipends for research in the new branch of physics, but has also unleashed the imagination of many a more or less informed thinker about these matters. There is nothing wrong with speculation per se-in fact, the progress of science hinges upon it - but the physicist has a responsibility here. On the one hand, he must try to bring his science to the man in the street and sometimes speculation might help to spruce things up a little. On the other hand, the layman will not always be able to accurately distinguish between facts and speculation and might easily be swayed by the scientific expert—even if his expertise pertains to a different field of science.

Two distinguished players in the field of quantum information theory have recently produced a popular work on quantum information. First, we will consider the book "Decoding Reality: the universe as quantum information" by Vlatko Vedral. Then, we will review "Coming of Age with Quantum Information," a book written by Christopher Fuchs (which rhymes with "books").

In writing his book, Vedral seems to have had two goals. He wished to share his broad insight and his scientific enthusiasm with those of us who have not been trained in the field of quantum information. But, besides this noble aim of "bringing-science-to-the-common-man," the book has another, more ambitious aim: Vedral wants to convince the reader that the whole of modern-day philosophy should be recast in an information-theoretic mold. He even argues that this recasting will finally answer the most fundamental questions of the philosopher: what is the 
nature of reality and where does it come from? These bold words should put readers on their guard.

But, before we blame Vedral for aiming too high, we will take a closer look at the structure of his work. It consists of three parts comprising 12 chapters. These parts are preceded by a "prologue" and the book concludes with an "epilogue." In the prologue, the rather pretentious argument that was mentioned above is introduced and is followed by the remark that information can be found anywhere in nature. In the first part, after introducing Shannon's information theory, Vedral convincingly shows us in the course of the next four chapters how this theory can and should be applied to various fields of science-physics, biology, sociology, and economics. The second part of the book introduces the reader to the wonderful world of the quantum. Without going into mathematical detail, the reader is acquainted with the several counter-intuitive features of quantum mechanics that make quantum information theory so promising.

The end of the second part, a chapter on randomness and determinism, is where the science ends and where philosophy begins. For reasons that become clear in the third part of the book, Vedral introduces the reader to the philosopher of science Karl Popper. Popper refined the notion of verificationism that was entertained by the logical positivists. They held that proper science should be restricted to statements that can be verified. The larger part of astrology was thus demoted from being a science into mere storytelling. It was Popper's insight, however, that general statements could never be verified. He famously illustrated his point with his story of the swans: no matter how often we encounter a swan that is white, there can be no logical justification of the statement "all swans are white"-it is always possible that the next swan we encounter will be black. Popper set himself the task of finding a true "method of demarcation" enabling us to distinguish proper science from pseudoscience. Instead of verificationism, he proposed falsificationism. According to the new doctrine, a proper scientific statement should be accompanied by a method through which the statement can be falsified. The possibility of encountering a black swan that would contradict our statement makes the latter a scientific statement.

When Vedral subsequently uses this doctrine to plead for his quantum information interpretation of reality, he seems to be oblivious to the fact that later philosophers of science (such as Wesley Salmon) have argued that the doctrine of falsificationism lacks any rational basis for preferring one unrefuted statement over another, i.e., there can be no progress in Popperian science. What, precisely, is Vedral's statement? In the first chapter, he says that "This book will argue that information (and not matter or energy or love) is the building block on which everything is constructed." Add to that the fact that information can be created out of nothing and we can answer the question regarding the origin of the universe. However, information is a characteristic of an object. It cannot exist without this object. It is only in the last chapter that Vedral again addresses this matter. He states here that "Scientific knowledge proceeds via a dialog with Nature. We ask 'yes-no' questions through our observations of various phenomena. Information in this way is created out of no information." But, what Vedral says here is different from his earlier statement. Rather than saying that information can be created out of nothing, 
he merely holds that it can be created out of no information. This conforms to the earlier mentioned intuition that information needs some kind of carrier-it needs to be information about something.

In physics, we can always choose in which type of coordinates to describe a system. Whereas Cartesian coordinates suffice to represent a game of billiard, spherical coordinates are much better suited for the description of celestial mechanics. In a similar vein, a scientist has considerable freedom in the choice of a fundamental substance in the description of phenomena. Whether he assumes that ultimately everything is made of energy and matter is merely one of energy's manifestations or vice versa-as long as the mathematics remains unchanged he is free to choose any substance. Only when the fundamental nature of the substance is taken to be ontological (i.e., pertaining to reality), does he require further arguments to justify this step. As we have seen, Vedral assumes information to be this fundamental substance. As long as he uses this assumption in scientific explanations that is no problem. But, when Vedral assumes the guise of the philosopher, he should tread with more care.

Vedral's argument for the fundamental nature of information is that it can explain its own origin. On the contrary, we have concluded that it cannot. Therefore, there is no longer reason to think that information is fundamental. Then, why is Vedral convinced of this? In one of the opening chapters of the book, he uses a statement of the American sociologist Maslow to explain the human urge to use causality in any description of nature: "if your only tool is a hammer, every problem looks like a nail." I think this explains the inclination of Vedral-after years of doing research in quantum information theory-to recognize information as essential for the description of every branch of science.

In the last few chapters of his book, Vedral makes several references to the contents of eastern religions. For example, he claims that certain elements of his quantum information interpretation can already be found in ancient Hindu philosophy: "Advaita Vedanta—a Hindu philosophy that emphasizes the oneness of the universe. In this view our perception of separate entities is just an illusion." What does Vedral mean by such remarks? Surely, there is no proof for his statements in these vague ancient notions. Rather, it seems that Vedral wishes to prevent his readers from getting the impression that his view of reality is eccentric, by saying "look, thousands of years ago people believed the same things!"

$\mathrm{He}$ is not alone in this. One of the founders of the Copenhagen Interpretation, Niels Bohr, frequently alluded to Buddhist philosophy in order to illustrate his arguments. There is, however, a danger in such an approach. To start with, let me admit that I am not an expert on Hindu philosophy. But, the remark above shows that Vedral is not a specialist either. Advaita (Sanskrit for "not two") philosophy is not monistic, but rather "non-dualistic." In it, a very peculiar role is played by some form of "cosmic consciousness." It is doubtful whether Vedral wishes to endorse this role. Of course, most metaphors are not supposed to be taken literally, and certain elements of this Hindu philosophy no doubt conform to his interpretation. The metaphor suits Vedral's second goal-that of convincing the reader that everything is quantum information-but I think, it distorts his aim of "bringing- 
science-to-the-common-man." As such, the metaphor does a lot more harm than good, as it distracts the reader's attention from the central argument.

Every 10 years, a part of the forest near the Los Alamos laboratory in New Mexico is destroyed in a controlled fire in order to prevent a conflagration of uncontrollable magnitude. In the year 2000, firefighters lost control over such a decennial fire. The results were catastrophic: the fire reached the laboratory and over 400 families lost their homes. One of those families was that of the quantum physicist Christopher Fuchs. Besides a host of other things, he lost his entire library.

For Fuchs, this event emphasized the value of the traces that the contents of his library had left in his written correspondence. Although all his books and printed articles were lost, an imprint of it all was retained in the large heap of his emails. To preserve this heap, Fuchs decided to publish it. First, on archive.org (the internet archive of Cornell University, used all over the world for the publication of preprints) and afterward as a weighty reader at the Cambridge University Press.

The correspondence in the book is centered on one theme: quantum information. The work consists of 33 chapters, each of which is dedicated to a separate correspondent. Many of these are fellow scientists who have contributed-and still contribute - to the development of the field of quantum information theory. This offers us an exclusive insight into the coming of age of this field.

Interestingly, the correspondents are not all physicists or philosophers. There are also curious journalists, inquiring students, and even a high school friend. This is at the same time, a positive and a negative feature of the book. On the one hand, it shows us the very human side of this scientific development. But, on the other hand, it causes the degree of technicality of the letters to vary enormously: one moment we read about the various flavors of Canadian beer; the next about "a particular pure-state decomposition of the density operator." Everyone will find something of interest in this book; there is no type of audience that will appreciate it all.

The subtitle of Fuchs's book is "Notes on a Paulian idea." Wolfgang Pauli was an Austrian-American physicist who greatly contributed to the early development of quantum mechanics. Today, he is best known for the "Pauli exclusion principle," which was discovered by Pauli in 1925, but this is not the "Paulian idea" Fuchs refers to. The description of the "Paulian idea" to which Fuchs does refer is quoted in a letter to a Missouri physics professor, Greg Comer. These are the words Pauli wrote:

In the new pattern of thought, we do not assume any longer the detached observer, occurring in the idealizations of this classical type of theory, but an observer who by his indeterminable effects creates a new situation, theoretically described as a new state of the observed system. In this way, every observation is a singling out of a particular factual result, here and now, from the theoretical possibilities, thereby making obvious the discontinuous aspect of the physical phenomena.

Nevertheless, there still remains in the new kind of theory an objective reality, inasmuch as these theories deny any possibility for the observer to influence the results of a measurement, once the experimental arrangement is chosen. 
Fuchs does not attempt to hide his enthusiasm about this idea when he says: "Man o' man I like that." The Paulian idea, it seems, consists in a belief in an objective reality of which any observer is an inextricable part. In the introductory section to this review, we already encountered several ways in which the quantum formalism can be interpreted. Of great importance, here is the question where to place the observer (in our case: the scientist). In the Copenhagen interpretation, the wave function of a system only collapses when this system is observed (yet, it is not clear how to define observation), but there are also interpretations in which an observer does not play such a pivotal role. An example of such an interpretation is the "Everett-Interpretation." According to this view, the wave function never collapses. Rather, the world is split into an infinite multitude of "possible worlds" each with its own slightly different wave function.

In 2000, Fuchs wrote an article for "Physics Today" with the title "Quantum Theory Needs No 'Interpretation.'” In this article, he argues, together with Asher Perez (whom Fuchs calls the "godfather" of quantum information), that the various interpretations of the quantum mechanical formalism merely "give the illusion of a better understanding," but we do not need any interpretation "to make quantum mechanics a useful guide to the phenomena around us." Notwithstanding this critical view on interpretational matters, Fuchs's book is one long sequence of discussions on precisely these topics. The solution to this seeming paradox lies in the fact that scientists and philosophers have different goals when they choose between theories. The scientist is primarily interested in making predictions and conducting experiments to test these. As we have seen above, this gives him great freedom of interpretation. However, for the philosopher, the interpretation is very important, giving rise to the stream of discussions.

In his book, Fuchs repeatedly chides the adherents of the Bohmian view, the realistic interpretation of quantum mechanics. At first sight, this may seem odd. Does not Pauli himself state that there remains some "objective reality" in the new theory? It is rather the nature of this reality where the difference lies between Pauli and Bohm. Where Bohm held that it must be somehow possible to formulate a realistic theory in such a way that there is no special role for an observer, Pauli explicitly states that there can be no "detached observer," and hence the Bohmian view is wholly wrong.

Whether the books written by Vedral and Fuchs will make fascinating reading probably depends on the reader's expectations. To be introduced to the topic of quantum information, Vedral provides a nice start. In light of the philosopher, however, this work does not appear so agreeable. On the contrary, Fuchs's book is not of much value for a novice to this field. Only for those readers with at least some knowledge of modern physics, it paints an entertaining picture of Fuchs's academic world.

Open Access This article is distributed under the terms of the Creative Commons Attribution Noncommercial License which permits any noncommercial use, distribution, and reproduction in any medium, provided the original author(s) and source are credited. 